\title{
Role of surgery following neoadjuvant chemoradiation in patients with lymph node positive locally advanced esophageal adenocarcinoma: a national cancer database analysis
}

\author{
Hirva Mamdani ${ }^{1}$, Thomas Birdas ${ }^{2}$, Shadia I. Jalal ${ }^{3}$ \\ ${ }^{1}$ Department of Oncology, Barbara Ann Karmanos Cancer Institute, Wayne State University, Detroit, Michigan, USA; ${ }^{2}$ Department of Surgery, \\ Thoracic Division, Indiana University Melvin and Bren Simon Comprehensive Cancer Center, Indianapolis, Indiana, USA; ${ }^{3}$ Department of Internal \\ Medicine, Division of Hematology/Oncology, Indiana University Melvin and Bren Simon Comprehensive Cancer Center, Indianapolis, Indiana, USA \\ Contributions: (I) Conception and design: All authors; (II) Administrative support: SI Jalal; (III) Provision of study materials or patients: T Birdas; \\ (IV) Collection and assembly of data: T Birdas; (V) Data analysis and interpretation: All authors; (VI) Manuscript writing: All authors; (VII) Final \\ approval of manuscript: All authors. \\ Correspondence to: Hirva Mamdani, MD. Department of Oncology, Barbara Ann Karmanos Cancer Institute, Wayne State University, Detroit, \\ Michigan, USA. Email: mamdanih@karmanos.org.
}

\begin{abstract}
Background: Concurrent chemoradiation (CRT) followed by surgery is a standard of care for locally advanced esophageal adenocarcinoma. It remains unclear if surgery following CRT offers any meaningful survival benefit compared to CRT alone in patients with clinical N3 disease who are at the highest risk of developing distant disease relapse.

Methods: We conducted analysis of the National Cancer Database (NCDB) to compare overall survival (OS) of patients with locally advanced esophageal adenocarcinoma (cTanyN1-3M0 based on AJCC 7th staging system) who underwent CRT with or without surgery and analyzed outcomes based on the cN stage.

Results: 7,520 patients were included in the analysis-74.7\% had cN1 disease, $21.1 \%$ had cN2 disease, and $4.3 \%$ had $\mathrm{cN} 3$ disease. The median OS advantage offered by CRT followed by surgery was 22, 15.8, and 9.6 months compared to CRT alone in $\mathrm{cN} 1, \mathrm{cN} 2$, and $\mathrm{cN} 3$ patients, respectively. The 5 -year OS estimates in the surgical group were $36.9 \%, 31.6 \%$ and $15.9 \%$ in $\mathrm{cN} 1, \mathrm{cN} 2$ and $\mathrm{cN} 3$ groups, respectively.

Conclusions: Surgery following CRT in patients with locally advanced esophageal adenocarcinoma leads to improvement in OS, with the largest benefit noted in patients with $\mathrm{cN} 1$ and cN2 disease. Surgery following CRT also confers meaningful long-term survival advantage for a subset of $\mathrm{cN} 3$ patients.
\end{abstract}

Keywords: Esophageal adenocarcinoma; esophagectomy; lymph node staging; N3 disease; National Cancer Database (NCDB)

Submitted Jun 02, 2021. Accepted for publication Sep 29, 2021.

doi: 10.21037/jgo-21-314

View this article at: https://dx.doi.org/10.21037/jgo-21-314

\section{Introduction}

Esophageal cancer is the $7^{\text {th }}$ most common cancer and the $6^{\text {th }}$ leading cause of cancer related deaths worldwide (1). In 2021, esophageal cancer is estimated to account for 19,260 new cases and 15,530 deaths in the United States (2). While the incidence of squamous cell carcinoma (SCC) of the esophagus has declined in the US, the incidence of adenocarcinoma (AC) has been rising dramatically (3). The majority of patients with esophageal AC present with locally advanced disease with a 5 -year overall survival (OS) of $43 \%$ (4). Trimodality therapy with neoadjuvant concurrent chemoradiation (CRT) followed

^ ORCID: 0000-0003-3874-8703. 
by surgery, as established by the CROSS trial, is currently a standard of care approach for locally advanced esophageal AC (5). The CROSS trial showed that compared to surgery alone, neoadjuvant CRT followed by surgery leads to a higher rate of R0 resection and a superior 5 -yr OS (47\% vs. $33 \%$, median follow-up 84 months) $(4,5)$. Two-thirds of the patients on this trial had N1 disease, defined as involvement of regional lymph nodes based on the older TNM staging system, but the actual number of involved lymph nodes was not reported. The updated analysis of the study reported distant disease recurrence in $39 \%$ of patients who underwent neoadjuvant CRT followed by surgery, however, the rates of distant disease recurrence and 5-year OS specifically in patients with positive lymph nodes remain unknown (4).

The $7^{\text {th }}$ edition of the American Joint Committee on Cancer (AJCC) staging system released in 2010 and subsequently the $8^{\text {th }}$ edition released in 2017 refined the $\mathrm{N}$ staging based on the data suggesting prognostic importance of the number of involved lymph nodes rather than mere presence and location of the positive nodes (6-12). $\mathrm{N}$ status now defines $\mathrm{N} 1, \mathrm{~N} 2$, and $\mathrm{N} 3$ designations as involvement of $1-2,3-6, \geq 7$ regional lymph nodes, respectively. Although this revision allows better survival stratification, it complicates the interpretation and application of the results of the trials that employed the old staging system such as the CROSS trial. This renders it difficult to tease out whether surgery following CRT is the optimal treatment irrespective of the number of positive lymph nodes. Additionally, patients with clinical T1-4aN2M0, TanyN3M0, and T4bN0-2M0 disease are now classified as having stage IVA disease in the $8^{\text {th }}$ edition as opposed to stage III disease in older editions of AJCC staging system.

A retrospective study of 176 patients with locally advanced esophageal cancer who underwent surgery following neoadjuvant CRT showed inferior OS in patients with 4 or more positive lymph nodes compared to those with $0-3$ positive lymph nodes (13). In locally advanced nonsmall cell lung cancer, presence of multi-station mediastinal lymph node involvement is associated with a high risk of recurrence, and surgery has not been shown to offer a survival advantage (14). These patients are often treated with definitive CRT followed by immunotherapy (15). It remains unclear if surgery offers any additional survival advantage in patients with locally advanced esophageal AC with higher lymph node stage who are at a high risk of systemic recurrence. In this study, we attempted to answer this question utilizing the National Cancer Database (NCDB). We compared OS of patients with locally advanced esophageal AC who underwent neoadjuvant CRT followed by surgery versus CRT alone and analyzed outcomes based on the number of involved lymph nodes according to the revised staging system.

We present the following article in accordance with the STROBE reporting checklist (available at https://dx.doi. org/10.21037/jgo-21-314).

\section{Methods}

The NCDB is a comprehensive oncology surveillance program that captures approximately $70 \%$ of all new cancer diagnoses in the United States from more than 1,500 Commission on Cancer-approved centers. It is maintained collaboratively by the American Cancer Society and the Commission on Cancer of the American College of Surgeons. The NCDB was queried for locally advanced resectable esophageal cancer cases diagnosed between 2010 and 2014. Patients included were 18 to 90 years old and had cTanyN1-3M0 esophageal AC based on the $7^{\text {th }}$ edition of AJCC staging system. Patients who underwent curative intent treatment with neoadjuvant CRT (radiation dose more than 4,000 cGy) with or without any form of R0 surgery including partial or total esophagectomy with or without gastrectomy were included in the final analysis. The primary outcome of interest was OS, which was defined as the time from diagnosis to last contact or death. We excluded patients with unknown survival status or timing of specific therapies. Patients with missing data were also excluded from the analysis. Data collected on each patient included demographics, Charlson-Deyo Comorbidity Index, clinicopathologic tumor parameters, lymph node stage using the $7^{\text {th }}$ TNM staging system, surgical resection versus none, and the type of treatment facility (academic/ research versus non-academic/community). The NCDB file was provided to the authors for analytical purposes and was exempt from institutional review board review. The study was conducted in accordance with the Declaration of Helsinki (as revised in 2013).

\section{Statistical analysis}

Statistical analysis was performed with SPSS software, version 23. Comparisons of sociodemographic parameters as well as tumor and treatment characteristics by group were performed with chi-square or Fischer's exact tests. Survival analysis was performed using the Kaplan-Meier method, and the log-rank test was used to evaluate the differences between the different $\mathrm{cN}$ subgroups. OS for 
Table 1 Key baseline patient demographics, clinical and tumor characteristics

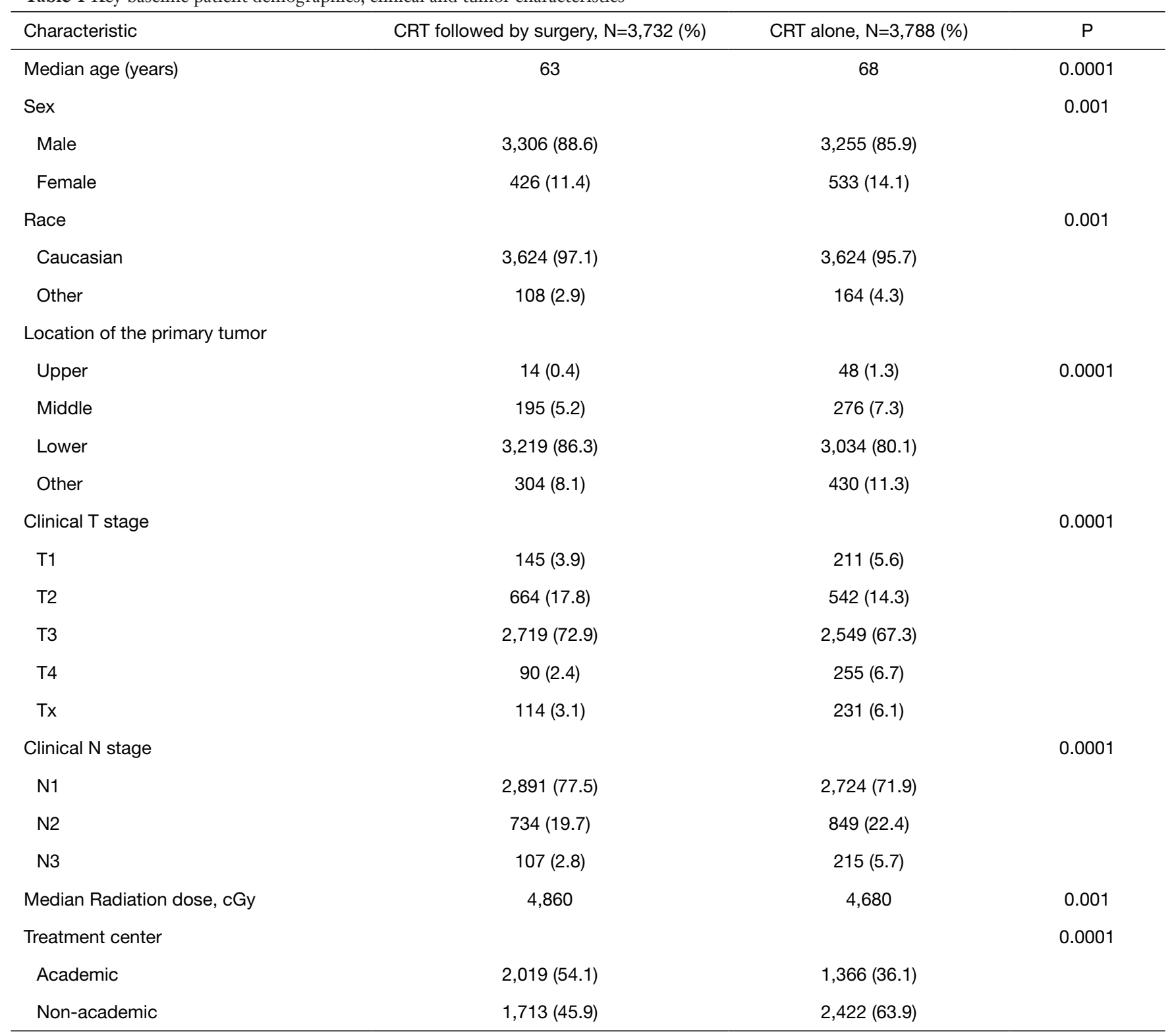

CRT, chemoradiation.

surviving patients was censored at the time of last contact. OS was compared between the surgical and non-surgical groups among the three different clinical $\mathrm{N}$ stages (cN1, $\mathrm{cN} 2$ and $\mathrm{cN} 3$ ). Multivariable analysis was performed with the Cox proportional hazards model, in order to study the effects of several prognostic factors on survival, expressed as hazards ratios (HRs); adjusted survival curves were generated for the overall population and the $\mathrm{cN}$ subgroups. $\mathrm{P}$ values for differences were calculated, with a significance level of $\mathrm{P}<0.05$.

\section{Results}

\section{Patient and treatment characteristics}

A total of 7,520 patients met inclusion criteria and were included in the analysis. Key baseline patient characteristics are summarized in Table 1 . The median age was 65 years (range, 18-90 years) for the overall cohort. The majority of patients were Caucasian (96.4\%) and male (87.2\%). Most tumors were in the lower esophagus (83.2\%). Of the overall cohort, 5,615 (74.7\%) patients had cN1 disease, 1,583 
Table 2 Kaplan Meier survival analysis based on treatment approach in different $\mathrm{cN}$ stages

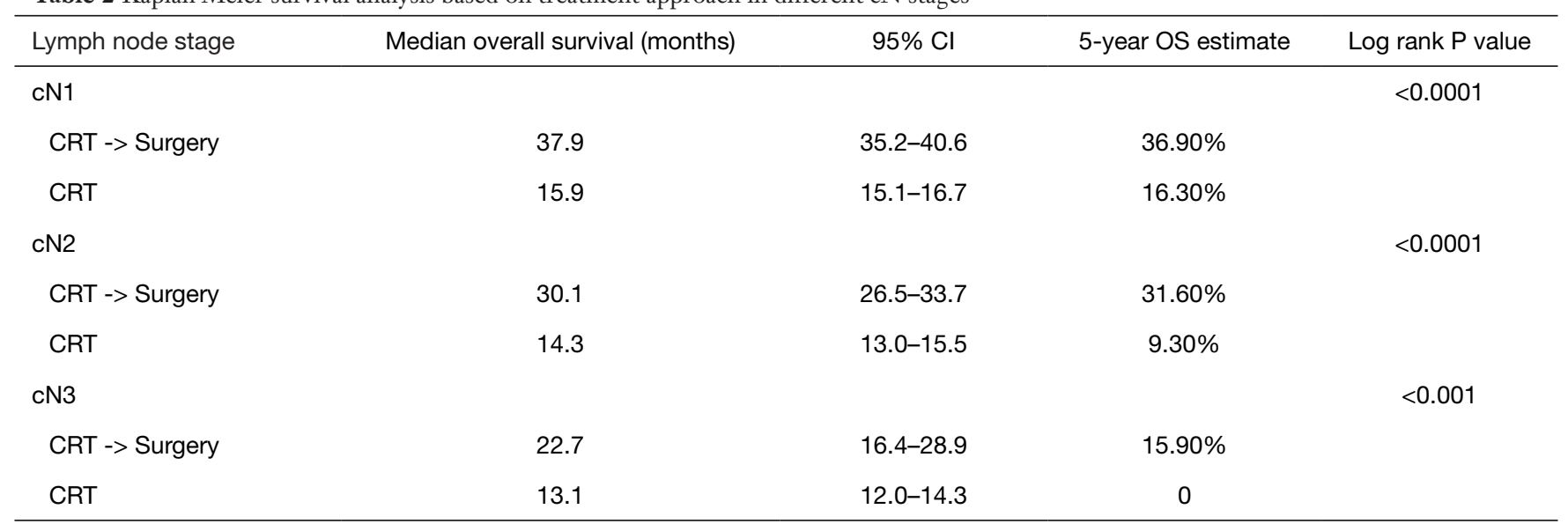

cN, clinical lymph node state; cN1, clinical N1; cN2, clinical N2; cN3, clinical N3; CRT, chemoradiation.

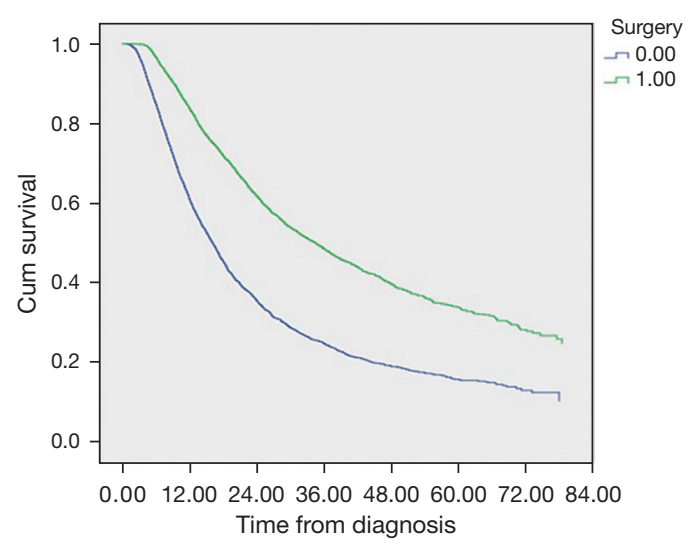

Figure 1 Overall Survival with CRT followed by surgery vs. CRT alone. Surgery 0.00: CRT alone without surgery, blue curve. Surgery 1.00: Surgery following CRT, green curve.

(21.1\%) had cN2 disease, and 322 (4.3\%) had $\mathrm{cN} 3$ disease. The majority of patients had cT3 disease as would be expected, and the percentage of patients with cT4 disease was slightly higher in the CRT group compared to CRT plus surgery group (6.7\% vs. $2.3 \%)$. A total of 3,732 patients $(49.6 \%)$ underwent surgery after neoadjuvant therapywith decreasing frequency in the higher nodal staging groups: $51.5 \%$ of $\mathrm{cN} 1$ patients, $46.4 \%$ of $\mathrm{cN} 2$ patients, and $33.2 \%$ of $\mathrm{cN} 3$ patients $(\mathrm{P}=0.0001)$. Median radiation dose was 4,860 cGy in CRT followed by surgery group and 4,680 cGy in the CRT alone group.

\section{Survival analysis}

The median follow-up was 19.6 months (range, 083.9 months). Five-year OS was superior among patients who received neoadjuvant CRT followed by surgery compared to CRT alone across all nodal groups (36.9\% vs. $16.3 \%$ in $\mathrm{cN} 1$; $31.6 \%$ vs. $9.3 \%$ in $\mathrm{cN} 2 ; 15.9 \%$ vs. 0 in $\mathrm{cN} 3$ group). Similarly, neoadjuvant CRT followed by surgery was associated with a longer median OS compared to CRT alone (mOS 37.9 vs. 15.9 months in $\mathrm{cN} 1, \mathrm{P}<0.0001 ; 30.1$ vs. 14.3 months in $\mathrm{cN} 2, \mathrm{P}<0.0001 ; 22.7$ vs. 13.1 months in $\mathrm{cN} 3$ group, $\mathrm{P}<0.001$ ) (Table 2). Figure 1 displays Kaplan-Meier survival curves for CRT followed by surgery versus surgery alone. In multivariate analysis female sex, younger age, higher income, care at an academic center, lower Charlson-Deyo score, lower $\mathrm{cN}$ status, lower $\mathrm{cT}$ stage, and surgical resection were independent predictors of long-term survival (Table 3).

\section{Discussion}

Neoadjuvant CRT followed by surgery is a standard of care for resectable locally advanced esophageal AC. With the refinement of the $\mathrm{N}$ classification to $\mathrm{N} 0-\mathrm{N} 3$ in the $7^{\text {th }}$ and subsequently $8^{\text {th }}$ edition of the AJCC staging system, $\mathrm{N}$ staging has become a valuable prognostic parameter. However, its implications in guiding treatment and determining the benefit of surgery remain unexplored. Therefore, examining these crucial questions using a large database such as the NCDB is of great value given the 
Table 3 Multivariable analysis of predictors of overall survival

\begin{tabular}{lcc}
\hline Variable & Hazard ratio (95\% Cl) & P \\
\hline Age & $1.009(1.006-1.013)$ & $<0.0001$ \\
Female sex & $0.90(0.82-0.98)$ & 0.006 \\
Income, highest quartile vs. lowest & $0.82(0.75-0.91)$ & $<0.0001$ \\
Charlson-Deyo Cl, $\geq 2$ vs. 0 & $1.30(1.14-1.48)$ & $<0.0001$ \\
Clinical T stage & $1.15(1.09-1.22)$ & $<0.0001$ \\
Clinical N stage & & $<0.0001$ \\
N2 vs. N1 & $1.19(1.11-1.28)$ & $<0.0001$ \\
N3 vs. N1 & $1.36(1.18-1.55)$ & 0.04 \\
Academic center & $0.94(0.88-0.99)$ & $<0.0001$ \\
Surgery & $0.49(0.46-0.53)$ & \\
\hline
\end{tabular}

substantial number of patients analyzed over the recent years.

The results of our study demonstrate that while addition of surgery to CRT offers OS benefit compared to CRT alone in patients with locally advanced esophageal AC, the survival difference narrows with the advancing $\mathrm{N}$ stage based on the number of involved lymph nodes. The OS of patients with $\mathrm{cN} 3$ disease is less compared to patients with $\mathrm{cN} 1$ or $\mathrm{cN} 2$ disease. This is not surprising as the higher nodal stage is associated with higher risk of micro-metastatic disease leading to distant recurrences. Esophagectomies can be associated with high mortality and morbidity (16). More than half of the patients experience compromised quality of life (QOL) from one or more therapy related long-term complications and it can take up to 9 months for the QOL to return to baseline postoperatively $(17,18)$. The prolonged recovery from surgery and associated decline in performance status could preclude a subset of patients from receiving systemic therapy if they were to develop recurrent disease shortly after surgery. While these considerations may imply that surgery may not offer meaningful benefit to patients with $\mathrm{cN} 3$ disease, our findings of 5 -year OS of nearly $16 \%$ with CRT followed by surgery versus $0 \%$ with CRT alone indicates that surgery following neoadjuvant CRT offers long term survival to at least a subset of patients with $\mathrm{cN} 3$ disease and should continue to be offered to these patients who are surgical candidates.

Future studies geared toward identification of predictive biomarkers of improved outcomes with trimodality therapy in this setting are needed to guide patient selection. Assessment of minimal residual disease following CRT and surgery utilizing circulating tumor DNA is one such example. Certain clinical factors that are known to be predictive of longer survival, including those identified in our study, may influence the decision making. For example, patients who are less likely to develop postoperative complications and consequent prolonged recovery including younger patients who are otherwise healthy with robust performance status and lower Charlson-Deyo score, and those who are being treated at an academic center experienced in esophagectomies may be appropriate surgical candidates despite having $\mathrm{cN} 3$ disease. Based on findings from retrospective studies showing no survival difference with the timing of esophagectomy following completion of CRT (within 8 weeks vs. after 8 weeks) (19), another reasonable approach might be to avoid immediate surgery in patients with extensive nodal disease with consideration of delayed esophagectomy if they do not present with widely metastatic disease. A PET/CT scan performed a few weeks after completion of CRT is valuable in identifying patients who develop disease progression during or shortly after receiving CRT. Finally, recently reported data on the use of adjuvant immune checkpoint inhibitor following trimodality therapy in locally advanced esophageal AC suggest that immune checkpoint inhibitors prevent some of the distant relapses and enhance the long-term survival offered by trimodality therapy even in patients with $\mathrm{cN} 3$ disease, which further supports the role of surgery in carefully selected patients with advanced nodal disease $(20,21)$.

Our study has several unavoidable limitations inherent to the studies utilizing a database or a registry. NCDB does not provide information on several salient patient-, tumor-, and treatment-specific factors such as details 
of chemotherapy regimen, toxicity, rate of pathologic complete response, pathologic staging, QOL, and patterns of recurrence, which limit the scope of current analysis. Additionally, non-randomized and retrospective nature of the study introduces an inherent selection bias. The database does not capture information on why a patient did not undergo surgical resection following CRT. Therefore, it is unclear whether the intent for these patients was to receive definitive CRT from the beginning or surgical resection was planned but not pursued because of factors that may confound OS analysis, such as disease progression during or shortly after completion of CRT, decline in performance status, or complications from CRT. Higher proportion of patients with tumors in upper one-third of the esophagus and a slightly higher proportion of patients with cT4 disease in CRT without surgery group argue towards the former case. Additionally, some patients with non-regional N3 disease or patients with $\mathrm{T} 4 \mathrm{~b}$ disease who may not be surgically resectable were still classified as having locally advanced disease and were treated with definitive intent CRT. Despite the limitations, the findings of this study are noteworthy and pave the way for prospective clinical trials to better define the subsets of patients with locally advanced AC who are most likely to benefit from surgery and those where immediate surgery following CRT should be avoided. The use of a national database with a large sample size and data on real world practice patterns provides a greater statistical power and makes the findings more generalizable.

\section{Conclusions}

The results of our study support the prognostic significance of nodal staging in patients with locally advanced esophageal $\mathrm{AC}$ and the importance of taking it into consideration when selecting patients for surgical treatment. In the absence of randomized prospective data, the results suggest that surgery following CRT confers an OS advantage compared to CRT alone, with the largest benefit noted in patients with $\mathrm{cN} 1$ and $\mathrm{cN} 2$ disease. Surgery following CRT also confers meaningful long-term survival advantage for a subset of $\mathrm{cN} 3$ patients. Consequently, surgery should be offered to eligible patients with cN1-3 disease following completion of CRT. Finally, with the introduction of immune checkpoint inhibitors in the adjuvant setting and emerging data on HER-2 directed therapy, the role of PD-L1 expression and HER-2 status, as well as detection of minimal residual disease in guiding decision on trimodality therapy in locally advanced disease remains to be determined.

\section{Acknowledgments}

Funding: None.

\section{Footnote}

Reporting Checklist: The authors have completed the STROBE reporting checklist. Available at https://dx.doi. org/10.21037/jgo-21-314

Peer Review File: Available at https://dx.doi.org/10.21037/ jgo-21-314

Conflicts of Interest: All authors have completed the ICMJE uniform disclosure form (available at https://dx.doi. org/10.21037/jgo-21-314). HM reports that she has received payment for participation in advisory board for Zentalis and AstraZeneca, as well as travel reimbursement from AstraZeneca for presentation of investigator-initiated trial in national research meeting. SIJ reports that she has received payment for participation in advisory board for Adaptimmune. She is the principal investigator of investigator-initiated trials with AstraZeneca, Astex, and Tesaro where the funding was provided to the institution. TB has no conflicts of interest to declare.

Ethical Statement: The authors are accountable for all aspects of the work in ensuring that questions related to the accuracy or integrity of any part of the work are appropriately investigated and resolved. The study was conducted in accordance with the Declaration of Helsinki (as revised in 2013). The NCDB file was provided to the authors for analytical purposes and was exempt from institutional review board review.

Open Access Statement: This is an Open Access article distributed in accordance with the Creative Commons Attribution-NonCommercial-NoDerivs 4.0 International License (CC BY-NC-ND 4.0), which permits the noncommercial replication and distribution of the article with the strict proviso that no changes or edits are made and the original work is properly cited (including links to both the formal publication through the relevant DOI and the license). See: https://creativecommons.org/licenses/by-nc-nd/4.0/. 


\section{References}

1. Global Cancer Observatory. International Agency for Research on Cancer, World Health Organization. Available online: https://gco.iarc.fr/. Accessed March 212021.

2. SEER Cancer Stat Facts: Esophageal Cancer. National Cancer Institute Surveillance, Epidemiology, and End Results Program. Accessed March 212021.

3. Thrift AP. The epidemic of oesophageal carcinoma: Where are we now? Cancer Epidemiol 2016;41:88-95.

4. Shapiro J, van Lanschot JJB, Hulshof MCCM, et al. Neoadjuvant chemoradiotherapy plus surgery versus surgery alone for oesophageal or junctional cancer (CROSS): long-term results of a randomised controlled trial. Lancet Oncol 2015;16:1090-8.

5. van Hagen P, Hulshof MC, van Lanschot JJ, et al. Preoperative chemoradiotherapy for esophageal or junctional cancer. N Engl J Med 2012;366:2074-84.

6. Rizk N, Venkatraman E, Park B, et al. The prognostic importance of the number of involved lymph nodes in esophageal cancer: implications for revisions of the American Joint Committee on Cancer staging system. J Thorac Cardiovasc Surg 2006;132:1374-81.

7. Rizk NP, Venkatraman E, Bains MS, et al. American Joint Committee on Cancer staging system does not accurately predict survival in patients receiving multimodality therapy for esophageal adenocarcinoma. J Clin Oncol 2007;25:507-12.

8. Peyre CG, Hagen JA, DeMeester SR, et al. Predicting systemic disease in patients with esophageal cancer after esophagectomy: a multinational study on the significance of the number of involved lymph nodes. Ann Surg 2008;248:979-85.

9. Mariette C, Piessen G, Briez N, et al. The number of metastatic lymph nodes and the ratio between metastatic and examined lymph nodes are independent prognostic factors in esophageal cancer regardless of neoadjuvant chemoradiation or lymphadenectomy extent. Ann Surg 2008;247:365-71.

10. Bogoevski D, Onken F, Koenig A, et al. Is it time for a new TNM classification in esophageal carcinoma? Ann Surg 2008;247:633-41.

11. Rice TW, Blackstone EH, Rusch VW. 7th edition of the AJCC Cancer Staging Manual: esophagus and esophagogastric junction. Ann Surg Oncol 2010;17:1721-4.
12. Rice TW, Patil DT, Blackstone EH. 8th edition AJCC/UICC staging of cancers of the esophagus and esophagogastric junction: application to clinical practice. Ann Cardiothorac Surg 2017;6:119-30.

13. Kesler KA, Helft PR, Werner EA, et al. A retrospective analysis of locally advanced esophageal cancer patients treated with neoadjuvant chemoradiation therapy followed by surgery or surgery alone. Ann Thorac Surg 2005;79:1116-21.

14. Martins RG, D'Amico TA, Loo BW Jr, et al. The management of patients with stage IIIA non-small cell lung cancer with N2 mediastinal node involvement. J Natl Compr Canc Netw 2012;10:599-613.

15. Antonia SJ, Villegas A, Daniel D, et al. Overall Survival with Durvalumab after Chemoradiotherapy in Stage III NSCLC. N Engl J Med 2018;379:2342-50.

16. Mamdani H KK, Jalal SI. Advances and Controvarsies in the Management of Locally Advanced Gastro-esophageal Adenocarcioma. J Clin Gastroenterol Treat 2016;2.

17. Scarpa M, Valente S, Alfieri R, et al. Systematic review of health-related quality of life after esophagectomy for esophageal cancer. World J Gastroenterol 2011;17:4660-74.

18. Sanghera SS, Nurkin SJ, Demmy TL. Quality of life after an esophagectomy. Surg Clin North Am 2012;92:1315-35.

19. Kim JY, Correa AM, Vaporciyan AA, et al. Does the timing of esophagectomy after chemoradiation affect outcome? Ann Thorac Surg 2012;93:207-12; discussion 212-3.

20. Kelly RJ, Ajani JA, Kuzdzal J, et al. Adjuvant Nivolumab in Resected Esophageal or Gastroesophageal Junction Cancer. N Engl J Med 2021;384:1191-203.

21. Mamdani H, Schneider B, Perkins SM, et al. A Phase II Trial of Adjuvant Durvalumab Following Trimodality Therapy for Locally Advanced Esophageal and Gastroesophageal Junction Adenocarcinoma: A Big Ten Cancer Research Consortium Study. Front Oncol 2021;11:736620.

Cite this article as: Mamdani H, Birdas T, Jalal SI. Role of surgery following neoadjuvant chemoradiation in patients with lymph node positive locally advanced esophageal adenocarcinoma: a national cancer database analysis. J Gastrointest Oncol 2021;12(5):1944-1950. doi: 10.21037/jgo21-314 